\title{
NDC-IVM: An automatic segmentation of optic disc and cup region from medical images for glaucoma detection
}

\author{
Umarani Balakrishnan \\ Department of ECE, Trichy Engineering College \\ Sivagnanam Nagar, Konalai, Trichy, Tamil Nadu 621132, India \\ umabkv@gmail.com
}

Received 5 October 2016

Accepted 16 January 2017

Published 8 March 2017

\begin{abstract}
Glaucoma is an eye disease that usually occurs with the increased Intra-Ocular Pressure (IOP), which damages the vision of eyes. So, detecting and classifying Glaucoma is an important and demanding task in recent days. For this purpose, some of the clustering and segmentation techniques are proposed in the existing works. But, it has some drawbacks that include inefficient, inaccurate and estimates only the affected area. In order to solve these issues, a Neighboring Differential Clustering (NDC) - Intensity Variation Masking (IVM) are proposed in this paper. The main intention of this work is to extract and diagnose the abnormal retinal image by identifying the optic disc. This work includes three stages such as, preprocessing, clustering and segmentation. At first, the given retinal image is preprocessed by using the Gaussian Mask Updated (GMU) model for eliminating the noise and improving the quality of the image. Then, the cluster is formed by extracting the threshold and patterns with the help of NDC technique. In the segmentation stage, the weight is calculated for pixel matching and ROI extraction by using the proposed IVM method. Here, the novelty is presented in the clustering and segmentation processes by developing NDC and IVM algorithms for accurate Glaucoma identification. In experiments, the results of both existing and proposed techniques are evaluated in terms of sensitivity, specificity, accuracy, Hausdorff distance, Jaccard and dice metrics.
\end{abstract}

Keywords: Glaucoma detection; optic disc; Gaussian mask updated; neighboring differential clustering; intensity variation masking; retinal image.

\section{Introduction}

Glaucoma is an optic nerve disease that is the major cause of blindness in worldwide. Generally, it is a disease of optic nerve that occurs due to the increased or decreased fluid pressure inside of the eye. ${ }^{1-3}$ The normal eye has the pressure of $21 \mathrm{~mm}$ of
$\mathrm{Hg}$, if it increases more than $21 \mathrm{~mm}$, the optic nerve will be damaged. Also, it permanently damages the vision and leads to the blindness if it is untreated. Classification $^{2,4}$ based on the Intra-Ocular Pressure (IOP) elevation is more useful in establishing the treatment for Glaucoma. Typically, there are

This is an Open Access article published by World Scientific Publishing Company. It is distributed under the terms of the Creative Commons Attribution 4.0 (CC-BY) License. Further distribution of this work is permitted, provided the original work is properly cited. 
different types of Glaucoma, which are illustrated as follows:

- Primary Open Angle Glaucoma

- Normal Tension Glaucoma

- Ocular Hypertension

- Primary Angle Closure Glaucoma

- Secondary Glaucoma

- Congenital Glaucoma

Moreover, Glaucoma ${ }^{5}$ is a silent disease that has no symptoms and warning. So, early detection of Glaucoma prevents from the permanent eye loss. The normal and Glaucoma affected eye is shown in Fig. 1.

\subsection{Problem identification}

Detecting glaucoma is an important and demanding task in medical image processing. For this purpose, various types of clustering and region prediction algorithms such as, level set method, ${ }^{6}$ Active Contour Model (ACM), ${ }^{7-9}$ fuzzy clustering ${ }^{10}$ and $k$-means clustering ${ }^{11,12}$ algorithms are proposed in the traditional works. Among these methods, then level set model performs the intensity and texturebased image segmentation processes by identifying the retinal optic disc in the image. The existing clustering and region-based methods are highly dependent on the observer's experience. These traditional techniques are not automated, which are manual, so the outcomes are based on the threshold value. Generally, the experts only know the exact threshold of manual detection process, which is denoted as the observer's experience. This is a major limitation of the existing techniques. Furthermore, most of the papers used the circular, elliptical and deformable models for disease identification.

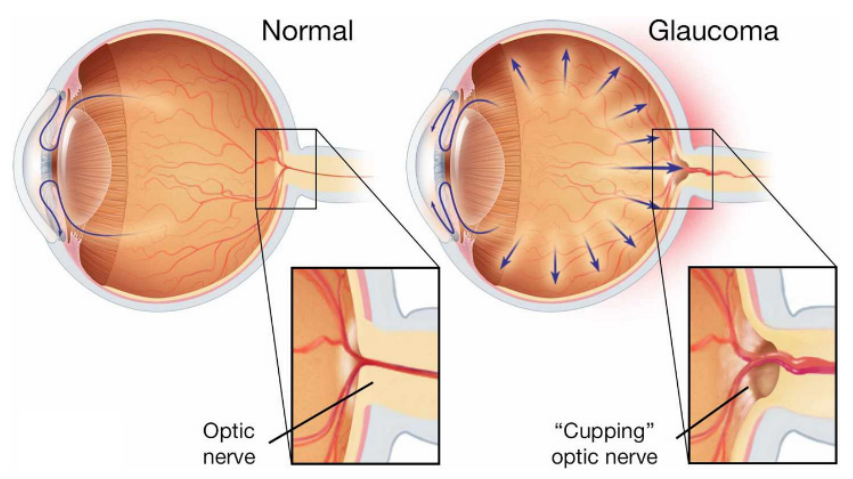

Fig. 1. Normal and glaucoma affected eyes.
Moreover, the existing techniques are time consuming, complex and not-suitable for day-to-day use. To solve these issues, a Neighboring Differential Clustering (NDC) with Intensity Variation Masking (IVM) methods are used in this paper for accurate Glaucoma detection and classification.

\section{Contribution}

The main contributions of this paper are as follows:

- The main aim of this work is to extract and diagnose the abnormality of retina by identifying the optic disc based on segmentation and clustering processes.

- To remove an additive noise and to provide smoothness to the image, a Gaussian Mask Updated (GMU)-based filtering technique is used.

- To predict the Region of Interest (ROI), the NDC clustering method is employed, which clusters the optic disc region and other retinal region.

- The novel concept of this paper are, it proposed a new clustering technique based on the distance between the nearest neighborhoods. Also, an intensity-based segmentation technique is developed for avoiding the misclassification by accurately identifying the location of optic disc and cup region.

\subsection{Organization}

The remaining sections of this paper are organized as follows: Section 2 presents the existing works that are relevant to Glaucoma identification and detection. Section 3 provides the detailed description about the proposed Glaucoma detection system. Section 4 evaluates the results of both existing and proposed techniques. Finally, the paper is concluded and the future work to be carried out is stated in Sec. 5 .

\section{Related Works}

This section presents some of the existing works related to detecting the abnormality in retinal image processing.

\subsection{Preprocessing}

Naz and $\mathrm{Rao}^{13}$ recommended different preprocessing techniques such as edge detection method, 
optimal thresholding method and manual threshold analysis for Glaucoma detection. In this paper, an ellipse filtering was applied to smooth the image and, to regulate the shape of segmented disc and cup boundary. Wankhade et al. ${ }^{14}$ proposed an algorithm to detect the retinal blood vessels for diagnosis. This includes the following stages:

- Preprocessing

- Image enhancement

- Feature extraction

- SVM classification

For efficient diagnosis, histogram equalization, binarization and wavelet transformation processes were applied in this work. Akram and $\mathrm{Khan}^{15}$ designed a computer aided system for early detection of Diabetic Retinopathy (DR). In this paper, the binary masks were created for noisy area and background, which was the combination of 1's and 0's. From this paper, it was analyzed that, it was important to determine both the foreground and background for abnormality detection. Jamal et al. ${ }^{16}$ developed an automated system for the diagnosis of DR eye disease. In this paper, the background estimation and noise removal processes were performed in the preprocessing stage for improving the quality of the image. Moreover, the local mean and variance were computed to remove the noise and to detect the background. Devi et al. ${ }^{17}$ extracted various wavelet features from three different filtering techniques that includes symlets (sym 3), daubechies (db 3) and biorthogonal (bio 3, bio 3.5 and bio 3.7) for Glaucoma detection. In this paper, the normal and Glaucoma images were classified with the help of energy signatures obtained from $2 \mathrm{D}$ discrete wavelet transform. Vijapur and Kunte ${ }^{18}$ suggested a pearson-R correlation filtering technique to detect Glaucoma. Here, the vertical diameters of optic-cup and disc were estimated to accurately segment the image.

\subsection{Segmentation and clustering}

Singh et $a .^{19}$ developed an automatic image processing technique for detecting Glaucoma from digital fundus images. In this paper, the wavelet feature extraction technique was integrated with the optimized genetic feature selection technique for diagnosis. It includes the following stages:

- Fundus image preprocessing

- Optic disc segmentation
- Wavelet feature extraction

- Feature selection using PCA

- Supervised classification

Here, a double windowing method was utilized to calculate the center of the optic disc. The major disadvantages of this paper were, it needs to improve both the accuracy and efficiency. Niwas et al. ${ }^{20}$ utilized the Minimum Redundancy Maximum Relevance (MRMR) and unsupervised Laplacian score feature selection algorithms for the detection of Angle Closure Glaucoma (ACG). The main intention of this paper was to segment the anterior part and to select the redundant features for machine classification. The major disadvantages of this method were, it was more expensive and required large memory space. Maheswari et al. ${ }^{21}$ proposed an Empirical Wavelet Transform (EWT) method for automatic diagnosis of Glaucoma. The Least Square-Support Vector Machine (LS-SVM) classification technique was utilized to classify the images as normal or Glaucoma. This work includes the following stages:

- Color extraction

- Feature extraction

- Feature ranking and normalization

- Classification using LS-SVM

The advantages of this paper were, it provided high performance, utilized minimum number of features, simple, fast and its performance do not depend on the image resolution. However, it does not utilize a large database for testing the proposed system, which was major drawback of this paper. Chen et $a .^{22}$ proposed an Automatic feature learning for Glaucoma Detection based on Deep LearnING (ALADDIN) technique Glaucoma diagnosis. In this paper, a contextualizing training strategy was utilized to learn the deep features of Glaucoma. Jayaram and Fleyeh ${ }^{23}$ surveyed the state-of-the-art in computational convex hulls in image processing. The major objectives of this paper were as follows:

- It explored the intricate applications

- It exploited the expanse of convex polygons

Samanta et $a l .{ }^{24}$ recommended a Back Propagation Neural Network (BPNN) classification technique for Glaucoma classification. This work contains the following stages:

- Image acquisition

- Feature extraction

- Image classification using BPNN 
The major advantages of this paper were, it utilized the features of the entire image without selecting the ROI, so it eliminated the complexity. Devasia et al. ${ }^{25}$ used a Particle Swarm Optimization (PSO) method for automatic optic disc localization and segmentation. In the preprocessing stage, resizing, blood vessels removal and red channel selection processes were performed. Then, the histogram equalization technique was applied to smooth the image. The PSO extracts the optic disc based on the global thresholding. Moreover, the publicly available dataset, namely, DRION was used to test the proposed system. Paul et al. ${ }^{26}$ proposed a Gaussian Mixture Model (GMM) to segment the Glaucoma from the Optic Coherence Tomography (OCT) images. In this paper, the ratio of the central portion of the optic nerve was estimated for Glaucoma diagnosis. Moreover, a curve partitioning method was applied to detect both the optic nerve and disc end points of the retinal and boundary. Mittapalli and kande $^{27}$ developed a novel blood vessel inpainting method to segment both the optic disc and cup from digital fundus images. The main intention of this paper was to detect the Glaucoma in the given image. Dutta et al. ${ }^{28}$ introduced an automatic image processing method to detect the diabetic retinopathy from fundus images. The main objective of this paper was to find the abnormalities in the image based on the location with its distance from macula. The advantage of this technique was, it significantly reduced the computational complexity.

\subsection{Classification}

Niwas et al..$^{29}$ introduced a fully automated method to detect the ACG abnormality from the OCT image. In this paper, the features from the raw Anterior Segment Optic Coherence Tomography (AS-OCT) images were extracted without parameter measurement and segmentation. It contains the following stages:

- Image acquisition

- Computed Hierarchy of Algorithm Representing Morphology (CHARM) features extraction

- Feature selection

- Multivariate classification

The major disadvantage of this paper was, the features extracted from the raw AS-OCT images were fully based on the compound image transformation. Moreover, the abnormality detection using manual labeling in huge ACG dataset was time consuming task. Niwas et al. ${ }^{30}$ suggested a waveletbased Local Binary Pattern (LBP) method for assessment of AS-OCT images. The major objective of this method was to collect the high quality AS-OCT images for the Glaucoma diagnosis. Here, the Naïve Bayes classification technique was applied to attain the final quality parameter. The main advantage of this method was, it selected the best image for ACG diagnosis with high accuracy. Issac et al. ${ }^{31}$ developed an adaptive threshold-based segmentation technique to improve the detection and classification of Glaucoma. This work comprises the following stages:

- Feature extraction

- Classification

In this work, two different techniques such as Support Vector Machine (SVM), Artificial Neural Network (ANN) were utilized for classification. Here, the Glaucoma was detected by considering the cup-to-disc ratio (CDR) and Neuro-Retinal Rim (NRR) area. However, it need to consider some alternate features of Glaucoma for improving the performance of detection, which was the main drawback of this method. Abirami and Shoba ${ }^{32}$ proposed a Fuzzy min-max Neural Network based on Data Core (DCFMN) technique for Glaucoma classification. This technique has three different layers that includes, input layer, middle layer and output layer. This work has the following stages:

- Color conversion, resizing and pruning

- Image enhancement

- Contrast adjustment

- Noise removal

- Segmentation

From this survey, it is analyzed that the existing segmentation and classification techniques efficiently detects the Glaucoma from the given image. But, it has some major drawbacks, which are clearly discussed in this section.

\section{Proposed Method}

This section presents the detailed description of the proposed Glaucoma detection and segmentation. At first, the given image is preprocessed to eliminate the noise by employing the GMU 
mechanism. Then, the cluster is formed in two ways, in the first way, the intensity difference is calculated. Then, the threshold is extracted, based on this value, the cluster is formed. In another way, the window is formed and the difference between the pixels is estimated for pattern extraction. Then, the weight of both clusters are calculated and it will be given as the input of segmentation. Here, the cup region and disc are segmented based on the ROI value. The flow of the proposed system is shown in Fig. 2, which contains the following stages:

- Preprocessing

- Clustering

- Segmentation

\subsection{Preprocessing}

The main intention of preprocessing the images is to improve the quality of the image by eliminating the noise and highlighting the features that are used in image segmentation. Before Glaucoma detection, the image must be preprocessed for getting a better quality. The most important problem in image processing is denoising, which is based on the features of the image. Moreover, it eliminates the noise and the edge blurring. In the existing works, there are many enhancement and preprocessing techniques including gray scale handling, histogram equalization and filtering for enhancing the quality of the image. The major drawback of the existing techniques are, a part of the noise still remains, threshold selection is a tough task, inherent reduction in overall image contrast and direct estimations are not accurate. Also, the low pass and high pass filtering techniques use the general format, so it may contain the blurred effect. Thus, the image must be tuned before processing it.

In order to solve these issues, a new technique, namely, GMU is used to preprocess the given image.

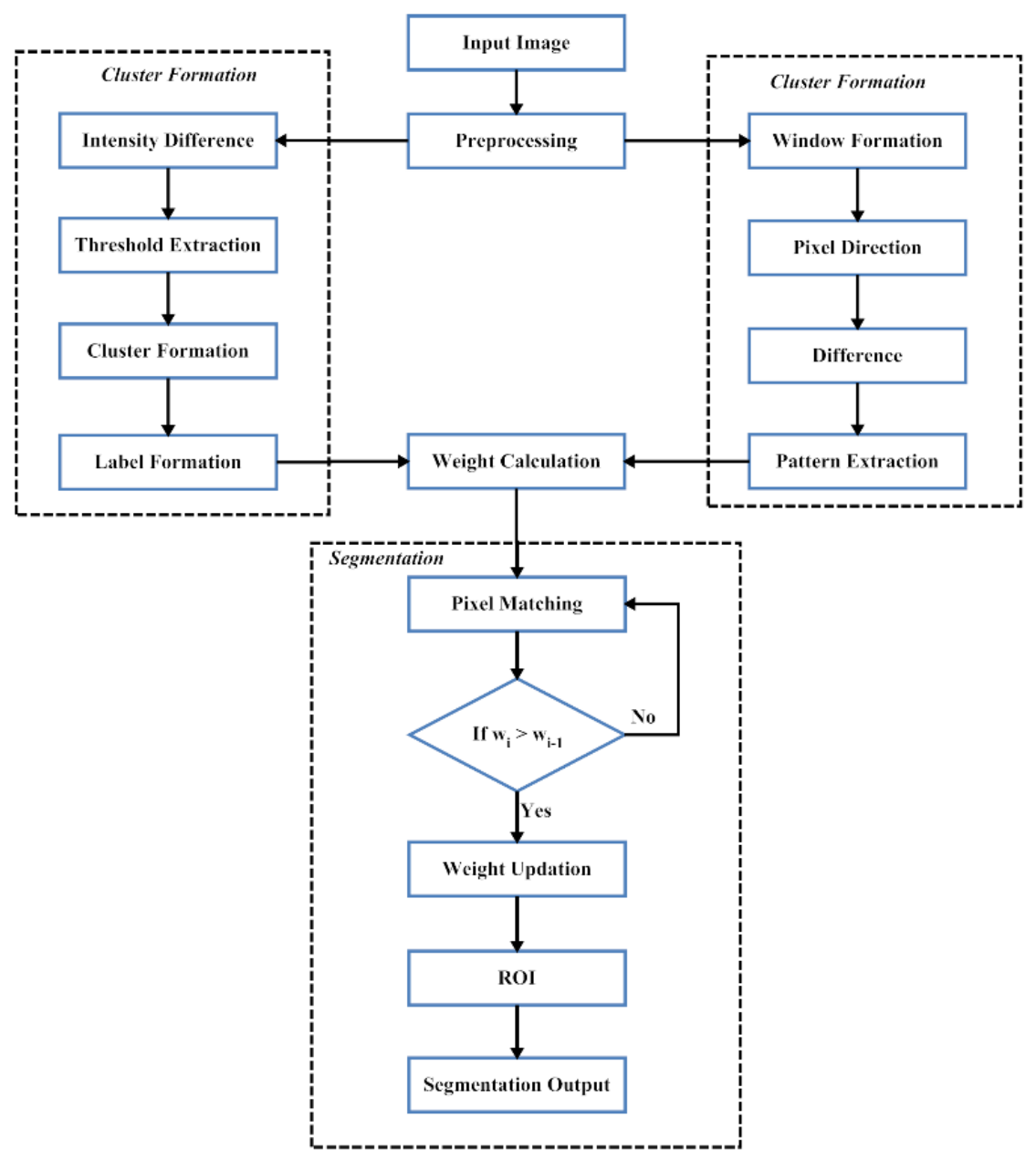

Fig. 2. Overall flow of the proposed system. 


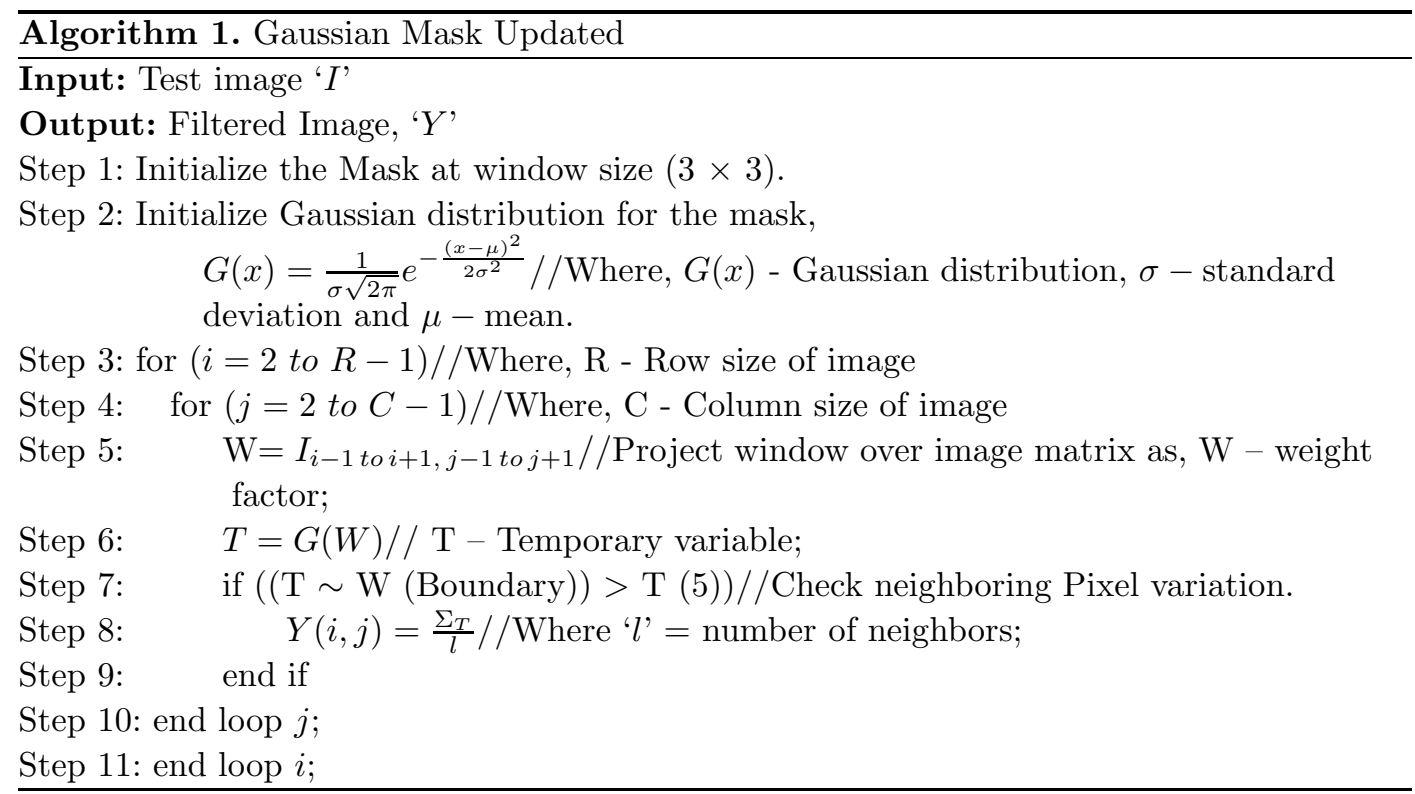

It eliminates the white Gaussian noise component and enhances the image quality for smoothing the image. In this technique, the losses are avoided by utilizing the value of neighborhoods. Also, it increases the effect of smoothening, so the image is preprocessed in a better quality. In Algorithm 1, the test image $I$ is given as the input and the mask is initialized at the window size of $(3 \times 3)$. Then, the Gaussian distribution of the mask $G(x)$ is initialized with the values of mean $\mu$ and standard deviation $\sigma$. Then, for each row and column, the window $W$ is projected over the image matrix. After that, the neighboring pixel variation is verified and if the center index of the value is greater than the weight boundary, the neighboring points of the filtered image is estimated. Figure 3(a) shows the original retinal image and Fig. 3(b) shows the output of preprocessed image.

\subsection{Clustering}

After preprocessing the image, the filtered image $Y$ is given as the input of clustering. Here, the cluster is formed based on the threshold and pattern extraction. In medical processing, clustering is defined as the process of organizing the image based on the

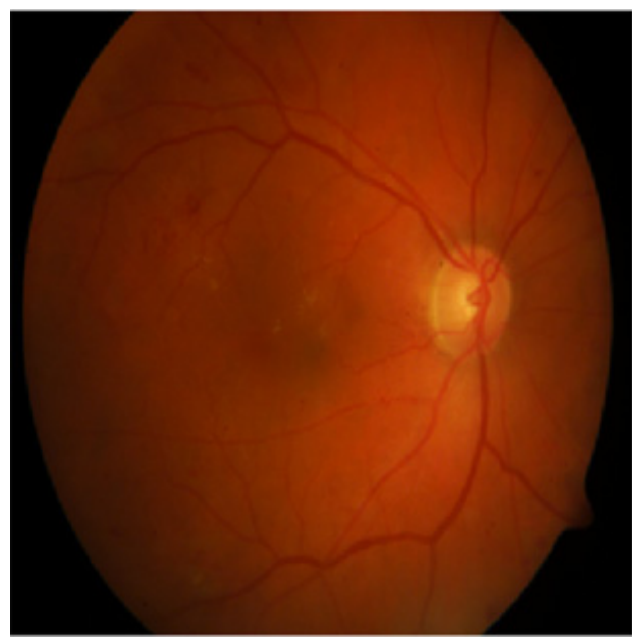

(a)

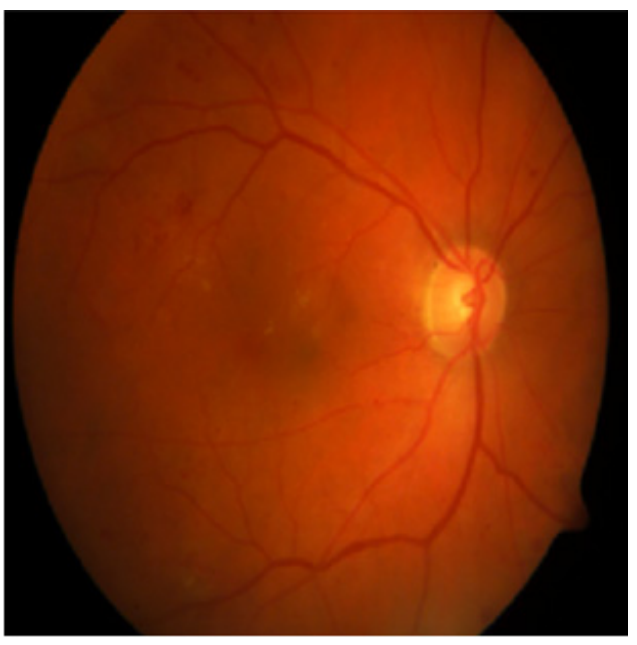

(b)

Fig. 3. (a) Input image and (b) preprocessed image. 
attributes or pixels. For clustering, the existing techniques such as Fuzzy C-Means (FCM), SelfOrganizing Map (SOM) and $K$-means are developed. But, it needs a threshold value and it requires to frame the rules for clustering. So, increases the time and memory complexity. To overcome these issues, this work developed a NDC technique for clustering the image. It finds the minimum and maximum differences between the neighbors to separate the histogram part. Based on this, the clustered output is obtained. The neighboringbased clustering technique fully works based on the concepts of mapping and distance. So, there is no need to fix the threshold value, this is the major advantage of the NDC technique. Based on the pattern recognition, the map result is updated and the map range is estimated by using the nearest neighbors. If the difference is greater than the map range, the system provides the clearance clustering result.

The main intention of clustering is to form a group of similar pixels in a specific region. In Algorithm 2, the maximum limit of image pixel intensity $\left(M_{p}\right)$ is initialized with the maximum and standard deviation of $Y$ coordinate point. Then, a Map window is created for clustering the image based on the range of $M_{p}$. The window coordinates $W_{x}$ and $W_{y}$ are estimated based on the value of $M_{w}$ and $Y$. After that, the radius value $R$ is initialized that represents the limit of moving window for updating the cluster weight $\left(C_{w}\right)$. It will be extracted by finding the exponential of $x, W_{x}, y$ and $W_{y}$. Then, the mapping window and radius values are updated, where the radius is updated for each $n$ number of iteration. The distance $D$ of the cluster weight is updated based on the intensity value. The minimum cluster index $(a, b)$ is extracted based on the distance value. The centroid of the cluster is estimated by varying the distance vector. After that, the indexed cluster is projected over the image for finding the best matching of image pixel with the cluster matrix. Finally, the clustered output is labeled in $C_{i j}$ and the iteration is repeated for each label updating.

The main advantages of the proposed NDC technique are as follows:

- It eliminates the noisy spots

- It obtains more homogeneous regions in the image

- It reduces the false blobs

- It is computationally faster

\subsection{Segmentation}

Segmentation is defined as the process of splitting an original image into digital images that is mainly used for object identification. It splits the object into set of pixels that are assigned different labels. The main aim of segmenting the given image is to modify the representation of image into meaningful. For segmentation, the PSO, blood vessel segmentation, edge growing, contour segmentation and thresholding techniques are developed in the existing work. But, it has some major drawbacks include: the mathematical formulation and high computation complexity. Also, inconsistent quality of segmentation, the limitation of regions mapping, and the locations are restricted. In order to solve these issues, this work proposed an IVM technique based on the cup region and the optic disc of the retinal image. Moreover, it locates the objects and boundaries based on the similarity and discontinuity of the pixels. It divided the entire image into subregions based on the common patterns. Also, it estimates the size of optical disc and exudates for clear location.

In Algorithm 3, the clustering image $C$ and filtered image $Y$ are given as the input. Here, the clustering constant $\alpha$, the inner weight $\beta$ and external weight $\gamma$ are initialized with the values of 0.2 and 1 . Then, the cluster coordinates $x_{i}, y_{i}$ and the binary mask $I_{x y}$ are initialized. The observation coefficient is extracted with the weight factor and it will be updated based on the value of cluster average. After that, the best light intensity is identified and the beta value is updated. The coordinates of $x$ position and $y$ position are also updated. The contour is applied for the value of $\beta_{x y}$ index. These steps are repeated until reaching the maximum iteration limit. Finally, the segmented output $S$ is estimated based on the updated mask value. Figure 4 shows the step by step output results of optic disc and cup region segmentation.

The major advantages of the proposed IVM segmentation technique are as follows:

- It is highly flexible that performs an automatic and interactive process for segmentation

- It generates clear object boundaries from the inner point to the outer regions

- It is efficient and fast for implementation

- It can be used in many real-time applications 
Algorithm 2. Neighboring Differential Clustering (NDC)

Input: Filtered image ' $Y$ '

Output: Clustered Image ' $C$ '

Step 1: Initialize maximum limit of image pixel intensity as, $M p=\{\max (Y), \operatorname{std}(Y)\} / /$

Where, $M p$ - Maximum limit;

Step 2: $M w_{i}=\frac{1}{1+e^{-Y_{N}\left(M P_{i}\right)}} \quad / /$ Where, ' $i$ ' $-1,2, \ldots, N, N$ represents the Size of Window.

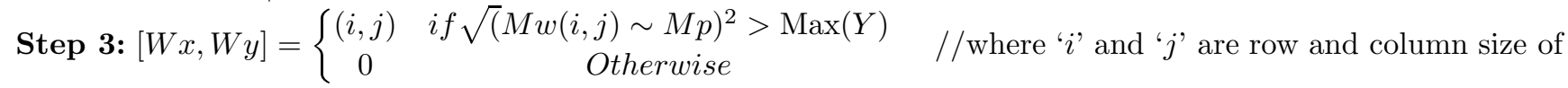
Map window.

Step 4: Initialize Radius, $R=\operatorname{size}(W x)$; / This radius represents limit of moving window for updating cluster weight.

Step 5: Cluster weight extraction,

$$
C w=R * e^{-\sqrt{(x-W x)^{2}+(y-W y)^{2}}} /(2 * R)
$$

Step 6: Update mapping window by,

$$
M w_{i, j}=M w_{i, j}+\left(C w * M w_{W x, W y}\right)-M w_{x, y}
$$

Step 7: Update the Radius of searching as,

$$
R=R+\left(\frac{\operatorname{Max}(M w)}{\operatorname{Max}(M w)+(E r * t)}-1\right) * \alpha
$$

Where, $\operatorname{Er}=(100-\operatorname{Max}(M w)), \alpha$ represents the cluster constant and $t$ represents the Number of iteration. Since the Radius value was updated for each ' $n$ ' number of iteration.

Step 8: Distance of cluster weight for change in intensity as,

$$
D=\sqrt{\left(M w_{i} \sim M w_{j}\right)^{2}}
$$

Step 9: Extract minimum cluster index of distance vector ' $D$ '

$$
[a, b]=\min (D)
$$

Step 10: Estimate centroid of cluster in varying Distance vector.

For $i=1$ to size $(M w)$

If $(\mathrm{a}>0 \& \& \mathrm{a}<\mathrm{R})$

$$
\begin{aligned}
& \operatorname{Cent}(i)=\frac{M w(i)+M w(b)}{2} \\
& \operatorname{Idx}(b)=0 \\
& D(b, i)=0 \\
& {[a, b]=\min (D)}
\end{aligned}
$$

End If;

End ' $i$ ' loop

Step 11: Project the indexed cluster over image and find best matching of image pixel with clustering matrix as,

$$
[\text { Vec, } \operatorname{Indx}]=\min \left(P(\mathrm{Cent})^{2}\right)
$$

Step 12: Labeled Cluster Output, $C_{i j}=Y(\operatorname{Indx})$

Step 13: Repeat Iteration for each label updation.

\subsection{Results}

This section evaluates the results of both existing and proposed techniques in terms of sensitivity, specificity, accuracy, jaccard, dice and Hausdorff distance. The existing techniques considered in this work are, Airpuff Intra-Ocular Pressure (IOP), super-pixel, Locally Linear Embedding (LLE), Sparse Coding (SC), Locality-constrained Linear Coding (LLC), Sparse Dissimilarity-constrained Coding (SDC) and ACM. The dataset used in this 


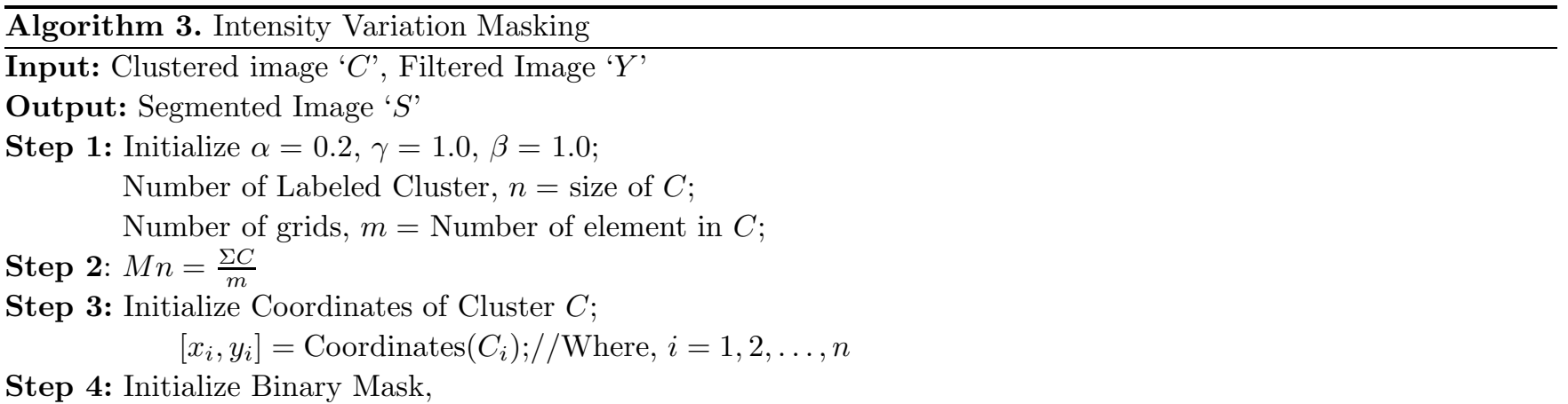

$$
I_{x y}= \begin{cases}1, & \text { if } C_{x y}==\operatorname{Max}(C) \\ 0, & \text { Else }\end{cases}
$$

Step 5: Extract observation coefficient, $\gamma=\sum_{i=1}^{n} w_{i} f+w_{0} ; / /$ Where, $w=\left[w_{0}, w_{1}, \ldots, w_{n}\right]$ represents the weight vector for Image Intensity $\mathrm{Y}$, $f$ represents the distance between nearest pixel intensity and $w$ can be estimate as,

$$
w_{i}=\sqrt{\left(Y_{0^{\circ}} \sim M n\right)^{2}+\left(Y_{90^{\circ}} \sim M n\right)^{2}}
$$

Step 6: Update Coefficient as,

$$
\gamma=\gamma \times \sqrt{\left(x_{i}-x_{j}\right)^{2}+\left(y_{i}-y_{j}\right)^{2}} ; / / \text { Where, } i=1,2,3, \ldots, n, j=1,2,3, \ldots, m ;
$$

Step 7: If $(I(i)<I(j)) / /$ Check best light intensity;

Step 8: $\beta_{x y}=1 * e^{\left(-\gamma * \gamma_{1}^{2}\right)} ; / /$ Update beta value;

Step 9: Update coordinates;

$$
\begin{aligned}
& x \text { position, } x_{n}=x_{n}(i) *(1-\beta)+x_{n}(j) * \beta+\alpha *(C(i)-M n), \\
& y \text { position, } y_{n}=y_{n}(i) *(1-\beta)+y_{n}(j) * \beta+\alpha *(C(j)-M n)
\end{aligned}
$$

Step 10: Apply Contour for the ' $\beta_{x y}$ ' Index

Step 11: Update Mask, $I$ as

$$
I_{x y}= \begin{cases}1, & \text { if } \beta_{x y}==1 \\ 0, & \text { Else }\end{cases}
$$

Step 12: Repeat Steps until maximum iteration limit.

Step 13: Segmented Output,

$$
S=I_{x y}
$$

work to evaluate the performance of all these techniques is Singapore Chinese Eye Study (SiMES). ${ }^{33}$

\section{(1) Dataset Description}

The SiMES dataset contains 2326 different subject eyes images, which are acquired by using the Canon-DGi and Canon CR-1 Mark-II digital retinal cameras. The dimension of the images are $3072 \times$ 2048 that have been manually measured by the trained professionals. In this dataset, there are 168 images which are diagnosed as glaucomatous by the ophthalmologists. Moreover, the SiMES dataset is randomly split into 325 are training images and 325 are testing disc images.

\section{(2) Sensitivity and Specificity}

In medical image processing, the sensitivity and specificity measures are widely used for diagnosis purpose. Sensitivity is defined as the true positive divided by the sum of true positive and false negative. Also, it is defined as the proportion of true positives that are correctly identified by the proposed NDC-IVM technique. Similarly, specificity is defined as the number of true negative results that are divided by the sum of the number of true negatives and false positives. The sensitivity and specificity are calculated as follows:

$$
\text { Sensitivity }=\frac{\mathrm{TP}}{\mathrm{TP}+\mathrm{FN}},
$$




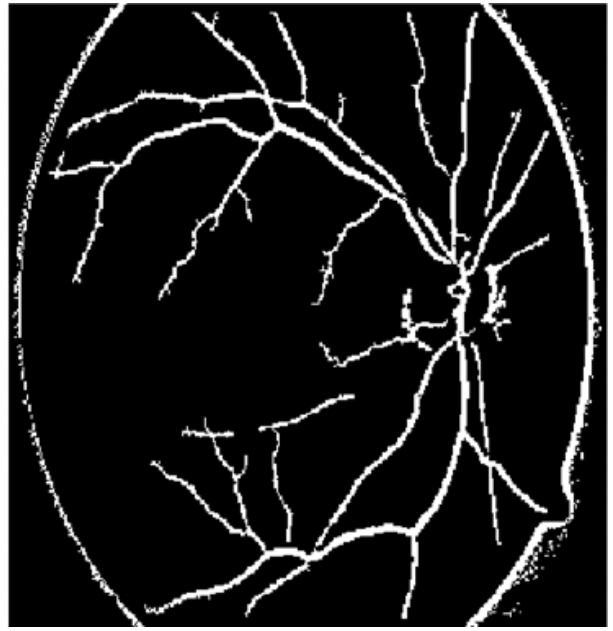

(a)

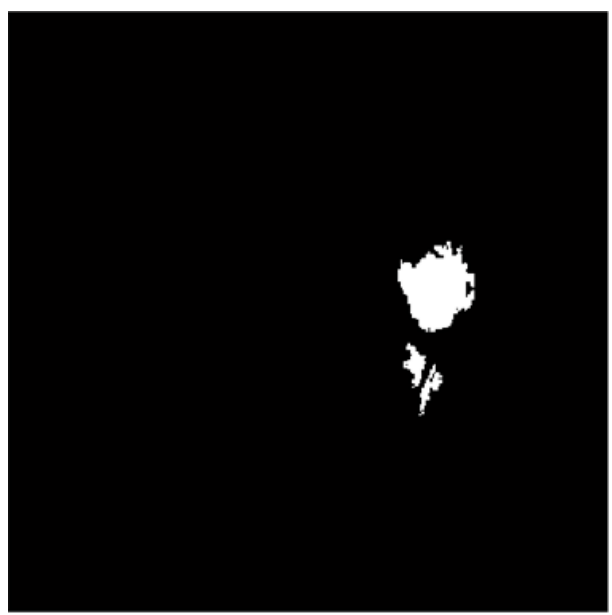

(c)

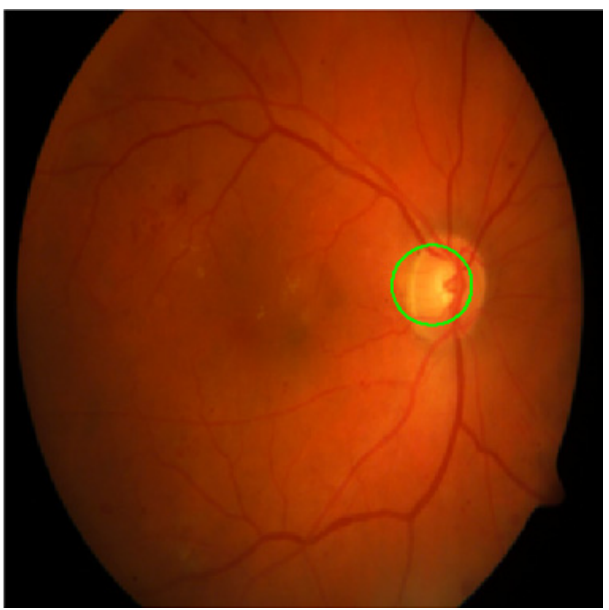

(e)

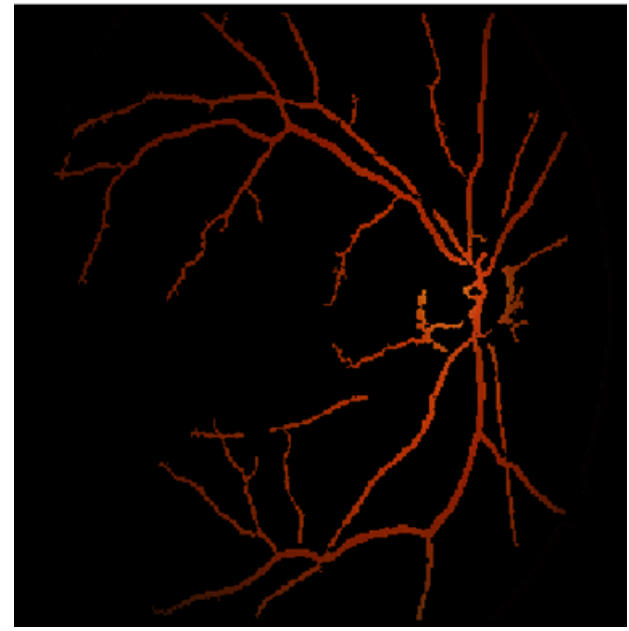

(b)

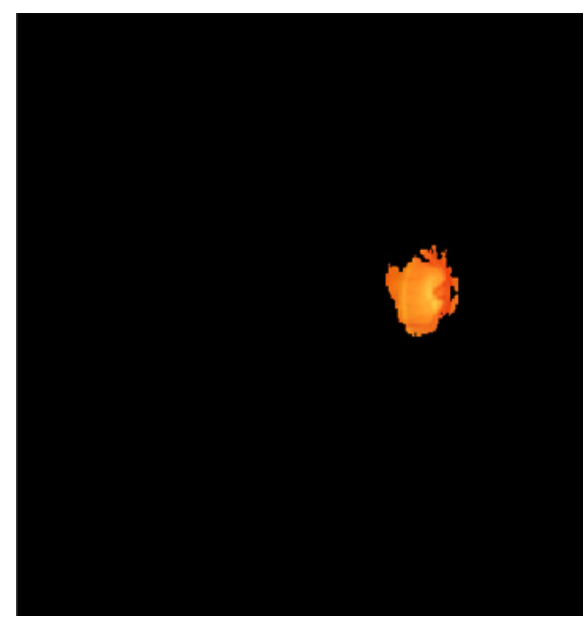

(d)

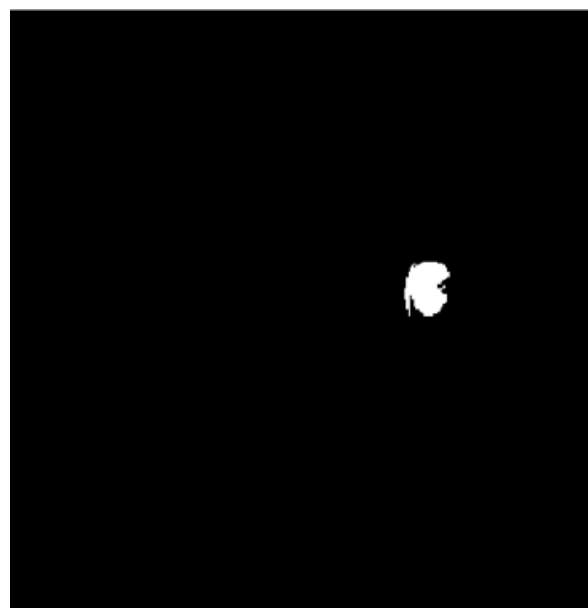

(f)

Fig. 4. (a) Blood vessel in binary representation. (b) Blood vessel extraction. (c) Optic disc binary representation. (d) Optic disc expected location. (e) Optic disc detection. (f) Cup region binary representation. (g) Cup region extraction and (h) Optic disc and cup region segmentation. 


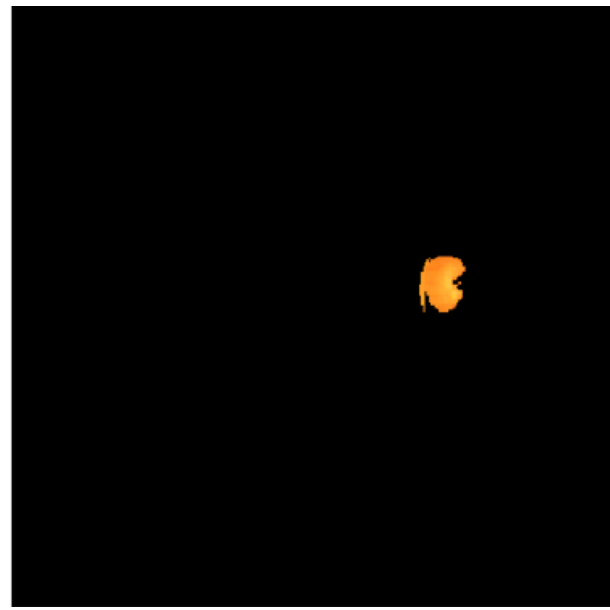

$(\mathrm{g})$

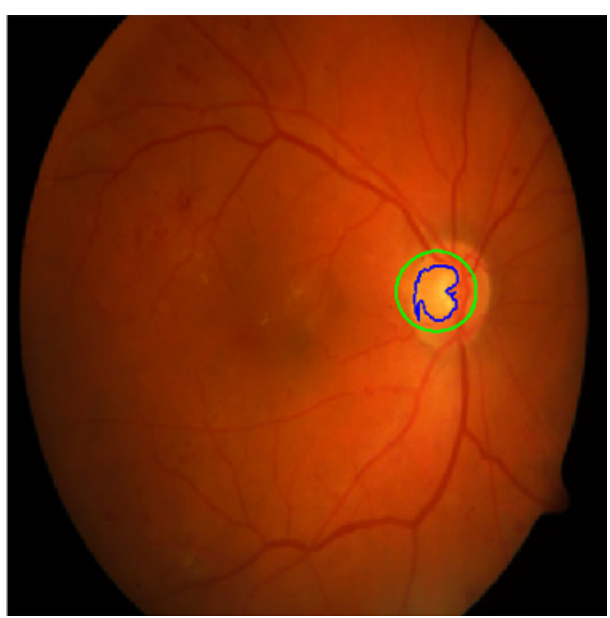

(h)

Fig. 4. (Continued)

$$
\text { Specificity }=\frac{\mathrm{TN}}{\mathrm{TN}+\mathrm{FP}},
$$

where, TP represents the true positive, FP represents the false positive, TN indicates the true negative and TP indicates the true positive. Figure 5 shows the sensitivity and specificity of the proposed NDC-IVM technique with respect to the number of segmented images. The terms used to define the sensitivity and specificity values are illustrated in Table 1.

The comparison between the existing and proposed techniques based on the sensitivity and specificity values are depicted in Fig. 6, where the $x$-axis

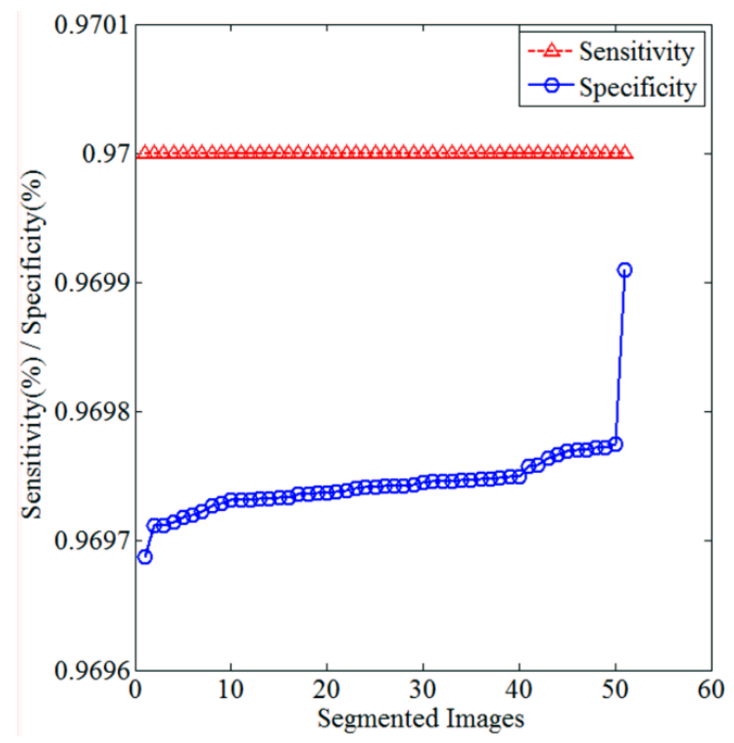

Fig. 5. Sensitivity and specificity. represents the specificity and $y$-axis represents the sensitivity. When compared the ACM model, the ROC of the proposed NDC-IVM is better with respect to the sensitivity and specificity values.

\section{(3) Accuracy}

The accuracy of the diagnostic test can measure how correct a diagnostic test identifies and excludes a given condition. It is determined from the sensitivity and specificity values, which is calculated as follows:

$$
\text { Accuracy }=\frac{\mathrm{TN}+\mathrm{TP}}{(\mathrm{TN}+\mathrm{TP}+\mathrm{FN}+\mathrm{FP})} .
$$

Figure 7 shows the accuracy of the proposed NDCIVM technique with respect to the number of segmented images.

\section{(4) Jaccard and Dice}

Jaccard and Dice are the similarity measures that are mainly used for diagnosis purpose in medical image processing. Jaccard is defined as the ratio between the intersection and union of two objects, then it varies from 0 to 1 . If the value is 1 , the two objects are identical and their sets have no common regions. Moreover, it finds the overlap between two labeled regions $r$ in $I_{1}$ and $I_{2}$ over the union. It is calculated as follows:

$$
\text { Jaccard }=\frac{\left|A_{r 1} \cap A_{r 2}\right|}{\left|A_{r 1} \cup A_{r 2}\right|} .
$$

To analyze the classification rate of the ground truth image, the Jaccard distance is used in this 
Table 1. Terms used to define sensitivity, specificity and accuracy.

\begin{tabular}{|c|c|c|c|}
\hline \multirow{2}{*}{$\begin{array}{l}\text { Outcome of the } \\
\text { diagnostic test }\end{array}$} & \multicolumn{3}{|c|}{ Condition determined by the standard of truth } \\
\hline & Positive & Negative & Row total \\
\hline Positive & $\mathrm{TP}$ & $\mathrm{FP}$ & $\begin{array}{l}\mathrm{TP}+\mathrm{FP} \text { (Total number of } \\
\text { subjects with positive result) }\end{array}$ \\
\hline Negative & $\mathrm{FN}$ & $\mathrm{TN}$ & $\begin{array}{l}\mathrm{FN}+\mathrm{TN} \text { (Total number of } \\
\text { subjects with negative test) }\end{array}$ \\
\hline Column total & $\begin{array}{l}\mathrm{TP}+\mathrm{FN} \text { (Total number of } \\
\text { subjects with given condition) }\end{array}$ & $\begin{array}{l}\mathrm{FP}+\mathrm{TN} \text { (Total number of subjects } \\
\text { without given condition) }\end{array}$ & $\begin{array}{l}\mathrm{N}=\mathrm{TP}+\mathrm{TN}+\mathrm{FP}+\mathrm{FN} \\
\text { (Total number of subjects in } \\
\text { study) }\end{array}$ \\
\hline
\end{tabular}

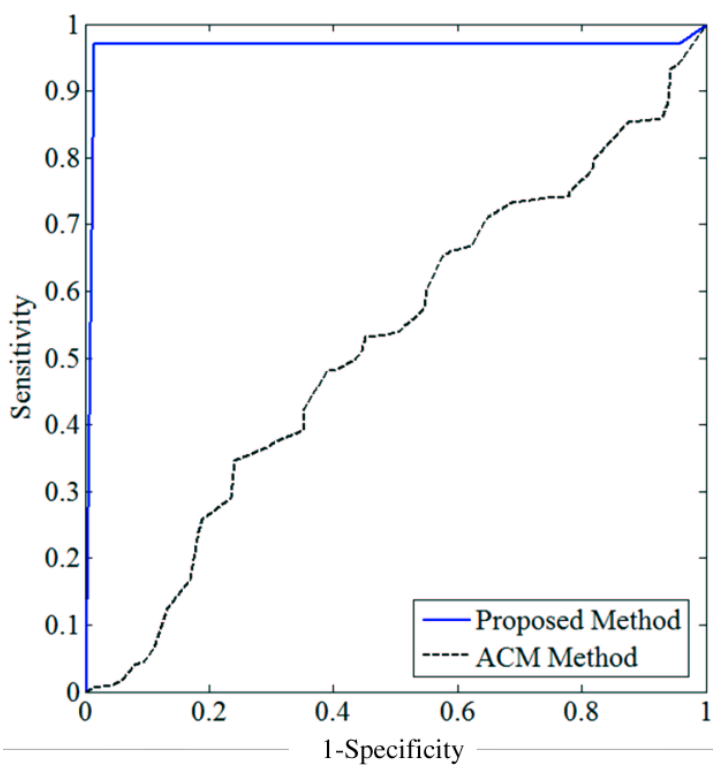

Fig. 6. ROC curve.

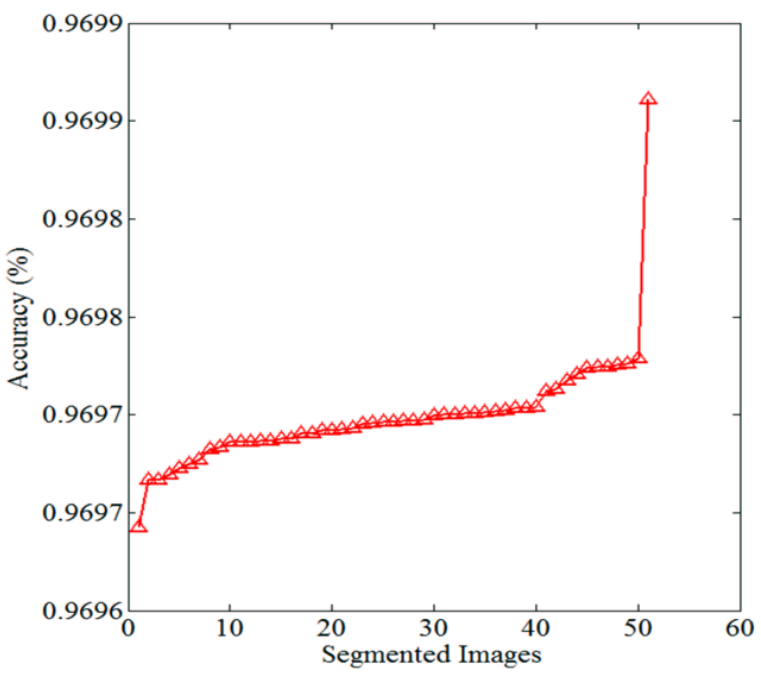

Fig. 7. Accuracy. work. Also, it is used to predict the outcome of the intersection ratio. Figure 8 shows the Jaccard and Dice values of the proposed technique, where the $x$-axis represents the number of segmented images and the $y$-axis represents the Jaccard/Dice similarity measure. Dice is a similarity measure that finds the similarity between two different images $A_{1}$ and $A_{2}$, which is calculated as follows:

$$
D_{s}=\frac{2 .\left|A_{r 1} \cap A_{r 2}\right|}{\left|A_{r 1}\right|+\left|A_{r 2}\right|} \text {. }
$$

\section{(5) Hausdorff Distance}

Hausdorff distance is a mathematical construct that measures the closeness of two sets of points, which are the subsets of a metric space. It is mainly used to find the degree of resemblance between two objects. Figure 9 shows the Hausdorff distance of the proposed system, where the distance is calculated based on the number of segmented images.

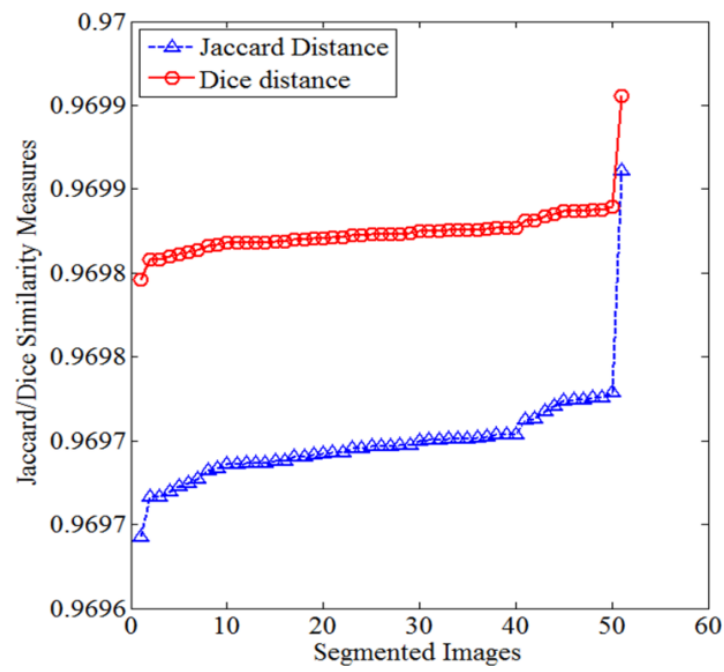

Fig. 8. Jaccard and Dice. 
NDC-IVM: An automatic segmentation of optic disc and cup region

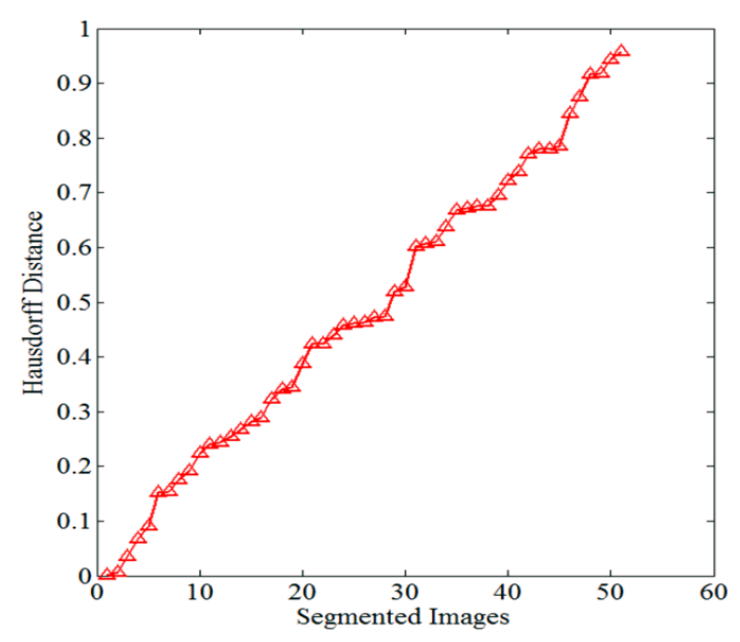

Fig. 9. Hausdorff distance.

In this analysis, the Hausdorff distance is mainly used to check the relevancy between the ground truth image and the segmented output image. If the relevancy level is high, it provides an accurate matching results.

\section{(6) Comparative Analysis}

Table 2 compares the performance of both existing $^{33}$ and proposed techniques based on Cup to Disc Ratio (CDR) error, pearson correlation and Area Under Curve (AUC). When compared to these existing techniques, the proposed NDC-IVM provides the better results. The larger CDR indicates the high risk of Glaucoma that is computed based on the ratio between the vertical cup diameter to the disc diameter clinically. Then, the pearson correlation measures the linear correlation between the manual and automated CDR. The AUC is the overall measure of the diagnostic strength of a test. Moreover, it is smaller during the diagnostics

Table 2. Performance analysis for SiMES dataset.

\begin{tabular}{lccl}
\hline Methods & CDR error & Pearson correlation & AUC \\
\hline Airpuff IOP & - & - & 0.59 \\
ACM & 0.121 & 0.351 & 0.628 \\
ASM & 0.117 & 0.328 & 0.613 \\
Level set & 0.124 & 0.447 & 0.68 \\
Superpixel & 0.078 & 0.59 & 0.8 \\
LLE & 0.08 & 0.47 & 0.75 \\
SC & 0.071 & 0.59 & 0.8 \\
LLC & 0.072 & 0.6 & 0.81 \\
SDC & 0.064 & 0.67 & 0.83 \\
NDC-IVM & 0.062 & 0.68 & 0.85 \\
\hline
\end{tabular}

Table 3. Comparison between existing and proposed techniques.

\begin{tabular}{|c|c|c|c|c|}
\hline Parameters & $\begin{array}{c}\text { Active } \\
\text { contour } \\
\text { model }(\mathrm{ACM})\end{array}$ & ASM & Level set & NDC-IVM \\
\hline True positive & 978 & 974 & 992 & 1007 \\
\hline True negative & 482 & 391 & 401 & 510 \\
\hline False positive & 64 & 92 & 82 & 36 \\
\hline False negative & 83 & 150 & 132 & 54 \\
\hline Accuracy & 0.9085 & 0.8494 & 0.8668 & 0.944 \\
\hline Sensitivity & 0.9218 & 0.9136 & 0.9236 & 0.9491 \\
\hline Specificity & 0.8828 & 0.7227 & 0.7523 & 0.9341 \\
\hline FRR & 0.1469 & 9.3342 & 8.2141 & 0.0957 \\
\hline FAR & 0.0614 & 5.725 & 5.1027 & 0.0345 \\
\hline
\end{tabular}

process. From this analysis, it is evident that the proposed NDC-IVM provides the minimized CDR error, high pearson correlation and AUC.

Table 3 compares the performance of existing $\mathrm{ACM}^{34}$ and proposed NDC-IVM models. The parameters considered for this analysis are, true positive, true negative, false positive, false negative, accuracy, sensitivity, specificity, False Acceptance Rate (FAR) and False Rejection Rate (FRR). From the results, it is analyzed that the proposed NDCIVM provides the better results, when compared to the ACM.

\section{Conclusion and Future Work}

This paper proposed an enhanced NDC-IVM-based clustering and segmentation techniques for Glaucoma identification from retinal images. The main aim of this paper is to accurately identify the cup region and optic disc of the Glaucoma affected image. This work contains three stages: preprocessing, clustering and segmentation. In preprocessing, the GMU technique is employed to eliminate the noise and to improve the visual quality of the image. Then, the filtered image is clustered based on the pattern and threshold extraction processes by using the proposed NDC clustering technique. After that, the pixel matching and weight updation processes are performed to segment the image. For this purpose, the IVM technique is used to accurately segment the cup region and optic disc from the given retinal image. The performance of both existing and proposed NDC-IVM techniques are evaluated in terms of sensitivity, specificity, accuracy, jaccard, dice, Hausdorff distance, CDR, pearson correlation 
and AUC. To evaluate the performance of these techniques, the SiMES dataset is utilized. In this evaluation, it is proved that the proposed NDCIVM outperforms the other techniques.

\section{References}

1. B. N. Kumar, R. Chauhan, N. Dahiya, Detection of Glaucoma using image processing techniques: A review, in microelectronics, IEEE 2016 Int. Conf. Computing and Communications (MicroCom). Durgapur, India.

2. M. S. Haleem et al., "Automatic extraction of retinal features from colour retinal images for glaucoma diagnosis: A review," Comput. Med. Imaging Graph. 37(7), 581-596 (2013).

3. D. D. Patil, R. Manza, G. C. Bedke, "Diagnose Glaucoma by proposed image processing methods," Int. J. Comput. Appl. 106(8) (2014).

4. K. P. Noronha et al., "Automated classification of glaucoma stages using higher order cumulant features," Biomed. Signal Process. Control 10, 174-183 (2014).

5. I. C. Campbell et al., "Automated segmentation of the lamina cribrosa using Frangi's filter: A novel approach for rapid identification of tissue volume fraction and beam orientation in a trabeculated structure in the eye," J. R. Soc. Interface 12(104), 20141009 (2015).

6. G. Jayanthi, G. M. A. Sagayee, S. Arumugam, "Glaucoma detection in retinal image using medial axis detection and level set method," Int. J. Comput. Appl. 93(3), 42-48 (2014).

7. M. C. V. S. Mary et al., "An empirical study on optic disc segmentation using an active contour model," Biomed. Signal Process. Control 18, 19-29 (2015).

8. S. Niu et al., "Robust noise region-based active contour model via local similarity factor for image segmentation," Pattern Recognit. 61, 104-119 (2017).

9. A. Mondal, S. Ghosh, A. Ghosh, Robust global and local fuzzy energy based active contour for image segmentation, Appl. Soft Comput. 47, 191-215 (2016).

10. K. Chaudhary, P. Maheshwari, S. Wadhwa, Glaucoma detection using cross validation algorithm: A comparitive evaluation on rapidminer, 2014 IEEE Conf. Norbert Wiener in the 21st Century (21CW). Boston, MA, USA.

11. M. R. K. Mookiah et al., "Automated glaucoma identification using retinal fundus images: A hybrid texture feature extraction paradigm," Image Anal. Model. Ophthalmol. 9-22 (2014).
12. J. Ayub et al., Glaucoma detection through optic disc and cup segmentation using $K$-mean clustering, 2016 Int. Conf. Computing, Electronic and Electrical Engineering (ICE Cube). Quetta, Pakistan.

13. S. Naz and S. N. Rao, "Glaucoma detection in color fundus images using cup to disc ratio," Int. J. Eng. Sci. 3, 51-58 (2014).

14. M. B. Wankhade, A. Gurjar, C. Sipna, "Detection of retinal blood vessels for disease diagnosis," Int. J. Eng. Sci. 3(3), 6-12 (2016).

15. U. M. Akram and S. A. Khan, "Automated detection of dark and bright lesions in retinal images for early detection of diabetic retinopathy," J. Med. Syst. 36(5), 3151-3162 (2012).

16. I. Jamal, M. U. Akram, A. Tariq, "Retinal image preprocessing: Background and noise segmentation," Telecommun. Comput. Electron. Control (TELKOMNIKA), 10(3), 537-544 (2012).

17. T. M. G. Devi, S. Sudha, P. Suraj, Glaucoma detection from retinal images, 2015 2nd Int. Conf. Electronics and Communication Systems (ICECS) (Coimbatore, India, 2015).

18. N. A. Vijapur, R. S. R. Kunte, Glaucoma detection by using Pearson-R correlation filter, 2015 Int. Conf. Communications and Signal Processing (ICCSP). Melmaruvathur, India.

19. A. Singh et al., "Image processing based automatic diagnosis of glaucoma using wavelet features of segmented optic disc from fundus image," Comput. Methods Programs Biomed. 124, 108-120 (2016).

20. S. I. Niwas et al., "Cross-examination for angleclosure glaucoma feature detection," IEEE J. Biomed. Health Inf. 20(1), 343-354 (2016).

21. S. Maheshwari, R. B. Pachori, U. R. Acharya, "Automated diagnosis of Glaucoma using empirical wavelet transform and correntropy features extracted from fundus images," IEEE J. Biomed. Health Info. (2016).

22. X. Chen et al., Automatic feature learning for Glaucoma detection based on deep learning, 18th International Conference Medical Image Computing and Computer-Assisted Intervention - MICCAI 2015, Munich, Germany, October 5-9, 2015, Proc. Part III, N. Navab et al., eds. (Springer International Publishing, Cham. 2015), pp. 669-677.

23. M. Jayaram, H. Fleyeh, "Convex hulls in image processing: A scoping review," Am. J. Intell. Syst. 6(2), 48-58 (2016).

24. S. Samanta et al., Haralick features based automated glaucoma classification using back propagation neural network, Proceedings of the 3rd International Conference on Frontiers of Intelligent Computing: Theory and Applications (FICTA) 2014 (Springer, 2015).

25. T. Devasia, P. Jacob, T. Thomas, "Automatic optic disc localization and segmentation using swarm 
intelligence," World Comput. Sci. Inf. Technol. J. 5(6), 92-97 (2015).

26. D. Paul, S. Priya, T. A. Kumar, GMM clustering based segmentation and optic nervehead geometry detection from OCT nervehead images, 2015 Global Conf. Communication Technologies (GCCT). Thuckalay, India.

27. P. S. Mittapalli, G. B. Kande, "Segmentation of optic disk and optic cup from digital fundus images for the assessment of glaucoma," Biomed. Signal Process. and Control 24, 34-46 (2016).

28. M. K. Dutta et al., "An efficient image processing based technique for comprehensive detection and grading of nonproliferative diabetic retinopathy from fundus images," Comput. Methods Biomechan. Biomed. Eng. Imaging Vis. 1-13 (2015).

29. S. I. Niwas et al., "Automated anterior segment OCT image analysis for Angle Closure Glaucoma mechanisms classification," Comput. Methods Programs Biomed. 130, 65-75 (2016).
30. S. I. Niwas et al., "Complex wavelet based quality assessment for AS-OCT images with application to Angle Closure Glaucoma diagnosis," Comput. Methods Programs Biomed. 130, 13-21 (2016).

31. A. Issac, M. Partha Sarathi, M. K. Dutta, "An adaptive threshold based image processing technique for improved glaucoma detection and classification," Comput. Methods Programs Biomed. 122(2), 229-244 (2015).

32. S. S. Abirami, S. G. Shoba, "Glaucoma images classification using Fuzzy Min-Max neural network based on data-core," Int. J. Sci. Mod. Eng. 1(7), 9-15 (2013).

33. J. Cheng et al., "Sparse dissimilarity-constrained coding for glaucoma screening," IEEE Trans. Biomed. Eng. 62(5), 1395-1403 (2015).

34. Y. Zhao et al., "Automated vessel segmentation using infinite perimeter active contour model with hybrid region information with application to retinal images," IEEE Trans. Med. Imaging 34(9), 1797-1807 (2015). 